\title{
Mass-Thickness Measurements in the TEM via EDS: A New Approach to Quantitative Chemical Analysis of Planetary Materials?
}

\author{
Thomas J. Zega ${ }^{1,2}$, Jane Y. Howe ${ }^{3}$, James Sagar ${ }^{4}$, Philippe Pinard ${ }^{4},{\text { Rachel } \text { Koch }^{3}}^{3}$ \\ ${ }^{1}$ Lunar and Planetary Laboratory, University of Arizona, Tucson, USA. \\ 2.Materials Science and Engineering, University of Arizona, Tucson, USA. \\ ${ }^{3}$ Hitachi High-Technologies America Inc., Clarksburg, USA. \\ 4. Oxford Instruments NanoAnalysis, High Wycombe, UK.
}

The chemical origins of our solar system are stored within meteorites and other planetary materials, the solid relics leftover from its birth 4.5 billion years ago. Energy-dispersive X-ray spectroscopy (EDS) has been central to compositional analysis of such materials. Coupled to an electron microscope, EDS can provide both qualitative and quantitative information in the form of false-color maps and as (normalized) elemental abundances, respectively. Both kinds of information are critical in planetary science for identifying two-dimensional compositional variations, and ultimately, as an aid to phase identification. Quantitative analysis is particularly important for planetary materials because it allows for comparison to equilibrium thermodynamic predictions and therefore constraining fundamental parameters, e.g., pressure and temperature, that are central to chemical models of the early solar nebula. Quantitative EDS has and will continue to be important for analysis of planetary materials including those we currently have in our collections and those to be returned by sample-return missions, e.g., Hayabusa 2 [1], OSIRIS-REx [2], and CAESAR [3] over the coming two decades.

In the transmission electron microscope (TEM), there are several approaches to quantitative EDS. For well over four decades, the 'Cliff-Lorimer (CL) approach' [4] has arguably been the most widely used method for quantitative EDS. By assuming a 'thin-foil criterion', i.e., that the sample is sufficiently thin so as not to absorb or fluoresce X-rays, the intensity of characteristic peaks are proportional to the elemental concentration multiplied by a detector sensitivity ' $k$ ' factor, determined by measuring reference standards. With sufficient counting statistics, $1 \%$ relative error is achievable. The CL approach is perfectly suitable for thin specimens but becomes problematic for thicker samples, where absorption and fluorescence can become significant. In comparison, the $\zeta$-factor method utilizes pure element thinfilm standards to derive a $\zeta$-factor assuming X-ray yield is proportional to mass thickness [5]. However, this approach requires measurement of beam current, which requires a sample holder with an integrated Faraday cup. Recently, [6] reported a new approach involving a single standard. Briefly, a thin film with known mass thickness serves as a reference without the need to measure beam current. This massthickness approach potentially offers a robust means of quantitative analysis without prior knowledge of beam current, and an alternative method to EELS for determination of sample thickness. Here we explore the mass-thickness approach to the analysis of $\mathrm{SrTiO}_{3}$ (STO) with the goal of applying it more broadly to planetary materials.

A $\mathrm{Si}_{3} \mathrm{~N}_{4}$ mass-thickness standard was used for the beam measurement, which establishes the expected X-ray yield under a standard set of optical and detector conditions. Our $\mathrm{Si}_{3} \mathrm{~N}_{4}$ sample was measured using a $200 \mathrm{kV}$ aberration-corrected Hitachi HF5000 S/TEM, recently installed at the University of

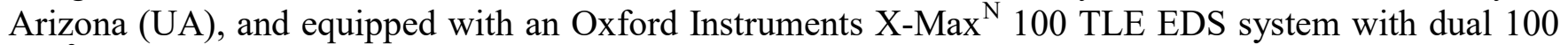
$\mathrm{mm}^{2}$ windowless silicon-drift detectors. The beam measurement was measured over a large $(>250 \mathrm{~nm})$ area and acquired until a total of 600,000 counts. The measurement was repeated to verify beam-current 
stability within $2 \%$ of the initial value. In addition to our $\mathrm{Si}_{3} \mathrm{~N}_{4}$ reference, we measured a sample of [100] $\mathrm{SrTiO}_{3}$ (purchased from Sigma Aldrich) prepared with an FEI Helios focused-ion-beam scanningelectron microscope (FIB-SEM) also at UA, using previously described techniques [7]. All spectra were acquired in scanning TEM (STEM) mode using a $35 \mu \mathrm{m}$ STEM condenser aperture (330 pA probe current) with the sample tilted $10^{\circ}$ clockwise about the sample-rod axis ( $\alpha$ tilt) toward the right detector (the left detector was turned off).

Figure 1 shows a reference spectrum from the STO sample. The major (characteristic) peaks of $\mathrm{Sr}, \mathrm{Ti}$, and $\mathrm{O}$ occur together along with $\mathrm{Cu}$ and minor $\mathrm{Fe}$. The $\mathrm{Cu}$ peak originates from the support grid that holds the FIB section, and the Fe is an artifact peak likely from the pole piece, but both were deconvoluted prior to quantitative analysis. We quantified the spectra using both the CL and massthickness approaches. Table 1 shows the quantification, in atomic $\%$, together with the deviation from ideal stoichiometry for both methods. We find that the mass-thickness approach quantifies the STO sample to within $0.1 \%, 3.7 \%$, and $4.0 \%$ of ideal stoichiometry for $\mathrm{O}$, $\mathrm{Ti}$, and $\mathrm{Sr}$, respectively. In comparison, the CL approach indicates significant deviations from stoichiometry. The STO sample was probed further using the mass-thickness approach, and we find that spatial variation in detector occlusion can be observed using this methodology which would have been overlooked using either CL or the $\zeta$-factor method. We hypothesize that this occlusion is due to differences in sample geometry between the $\mathrm{Si}_{3} \mathrm{~N}_{4}$ reference sample and the FIB section. We will discuss the implications of these differences at the meeting [8].

\section{References:}

[1] S Watanabe et al, Space Sci Rev 208 (2017), p. 3.

[2] DS Lauretta et al, Space Sci Rev (2017), p. 925.

[3] SW Squyres et al, Lunar \& Planet Sci Conf (2018) Abstract \#1332.

[4] G Cliff and GW Lorimer, Journal of Microscopy 103 (1975), p. 203.

[5] M Watanabe and DB Williams, Journal of Microscopy 221 (2006), p. 89.

[6] P Statham et al, IOP Conf. Ser.: Mater. Sci. Eng. 304 (2017), 012017

[7] TJ Zega et al, Meteoritics \& Planet. Sci. 42 (2007), p. 1373.

[8] We gratefully acknowledge NASA (grants \#NNX12AL47G and \#NNX15AJ22G) and NSF (grants \#1531243 and \#0619599) for funding of instrumentation in the Kuiper Materials Imaging and Characterization Facility at the Lunar and Planetary Laboratory, University of Arizona. Research supported by NASA grant \#NNX15AJ22G.

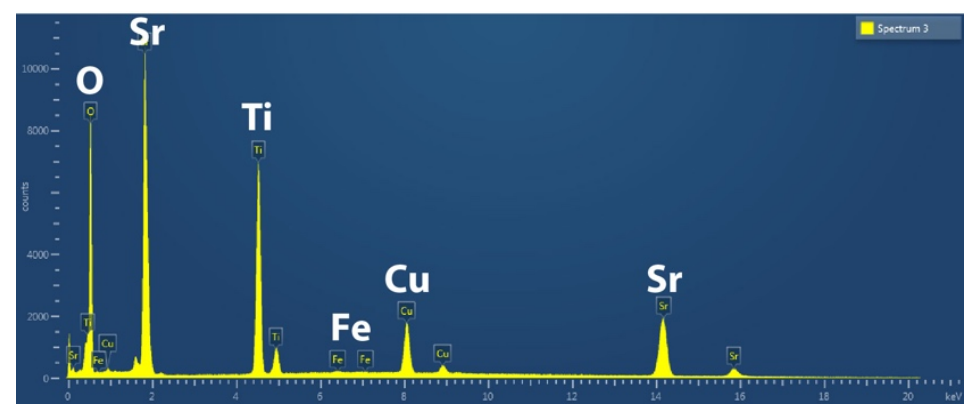

Figure 1 (left). EDS spectrum of STO. $\mathrm{Cu}$ and $\mathrm{Fe}$ are artifacts. Table 1 (below). Quantification of STO spectrum using mass-thickness (MT) and CL approach. Difference $(\delta)$, relative to stoichiometry, shown in percent.

\begin{tabular}{|c|c|c|c|c|}
\hline \multirow{2}{*}{ Table 1 } & \multicolumn{2}{|c|}{ Atomic \% } & \multicolumn{2}{c|}{$\delta$ (\%) rel. to stoich. } \\
\cline { 2 - 5 } & $\mathrm{MT}$ & $\mathrm{CL}$ & $\mathrm{MT}$ & $\mathrm{CL}$ \\
\hline $\mathrm{O}$ & 60.07 & 52.65 & 0.1 & 12.3 \\
$\mathrm{Ti}$ & 20.74 & 24.51 & 3.7 & 22.6 \\
$\mathrm{Sr}$ & 19.19 & 22.84 & 4.0 & 14.2 \\
\hline Total & 100 & 100 & \multicolumn{3}{|c|}{} \\
\hline
\end{tabular}

